\title{
Elastic Networks thematic network results I: planning and control of Flex-Grid/SDM
}

\author{
F.J. Moreno-Muro ${ }^{1}$, R. Rumipamba-Zambrano ${ }^{2}$, J. Perellón ${ }^{2}$, P. Pavón-Mariño ${ }^{3}$, J. Solé2, R. Martínez ${ }^{3}$, R. \\ Casellas $^{3}$, R. Vilalta ${ }^{3}$, R. Muñoz ${ }^{3}$, L. Ruiz ${ }^{3}$,J. Mata ${ }^{4}$, N. Merayo ${ }^{4}$, I. de Miguel ${ }^{4}$, R.J. Durán ${ }^{4}$ \\ ${ }^{1}$ Universidad Politécnica de Cartagena, ${ }^{2}$ Universitat Politécnica de Catalunya, ${ }^{3}$ Centre Tecnologic de \\ Telecomunicacions de Catalunya, ${ }^{4}$ Universidad de Valladolid
}

\begin{abstract}
This paper overviews the approach of the Elastic Networks research network to address different issues of planning and control of Flex-Grid/SDM optical networks. Firstly, we present the Net2Plan open-source planning tool capabilities to model Flex-Grid/SDM networks; secondly a PCE-based Transport-SDN controller for packet over flex-grid optical networks is described. Finally results on machine-learning-based QoT classification techniques useful in planning and control tasks are reported.
\end{abstract}

Keywords: Elastic optical networks, Space Division Multiplexing, network planning, control, machine-learning

\section{FLEX-GRID/SDM NETWORKING PROBLEMS WITH PLANNING TOOLS}

Space division multiplexing (SDM) allows to scale up the capacity of the current fiber systems, combined with the spectral elasticity of Flex-Grid technology enables transmissions at ultra-high bit rates coping with the IP traffic growth foreseen in the next years. Several works in the literature have investigated the joint FlexGrid/SDM view and driven possible switching schemes at the network nodes. In [2], authors propose a broadcast-and-select (B\&S) SDM-capable Reconfigurable Optical Add Drop Multiplexer (ROADM) to constrain the lightpaths to a single core along the whole path. This is called as the Core Continuity Constraint (CCC) and its performance, in a static scenario, is compared to the Fully-Non-Blocking (FNB) SDM-ROADM option. To this aim, an Integer Linear Programming (ILP) formulation has been developed for both SDMROADM types. An exhaustive extension of [2] is presented in [3], where the authors also compared the two SDM-ROADMs with a novel heuristic algorithm in two reference backbone topologies.

A common relevant point of both studies ([2] and [3]) is that they have been developed and tested in an opensource planning tool, namely, Net2plan [1]. Net2plan is a free and open-source Java tool devoted to the planning, optimization and evaluation of communication networks, which uses the open-source Java Optimization Modeler $(\mathrm{JOM})$ as an interface to external solvers, such as CPLEX or GLPK, in an easy way allowing users develop and testing their own formulation-based algorithms. In [2] and [3], Net2plan has also been used as a framework to model Flex-Grid/SDM networks, where the link capacity is measured in frequency slots (FSs) and a set of transponders are considered to satisfy the traffic demands along the reference topology. The transponders are mainly modelled by means of five parameters: (i) line rate in Gbps, (ii) cost, (iii) number of FSs to occupy in the links, (iv) optical transmission reach and (v) cost of the signal regenerator. The network model is aware of the Multi-Core Fibers (MCFs) by labelling with unique $i d$ the emulated cores in each link and assuming an intercore crosstalk (ICXT) that penalizes the optical reach depending on its modulation format. Fig. 1 depicts an example of using Net2plan as a framework to emulate a Flex-Grid/MCF network scenario. This snapshot of the planning tool has two differentiated parts; on the left side of Fig.1, the emulated network is modelled by a set of geo-positioned nodes interconnected by a set of MCF links. Furthermore, the table on the right shows the carried traffic using optimized paths (which include the sequence of core $i d s$ per lightpath and FS) to minimize the network CapEx. Such traffic requests are satisfied by a set of flexible transponders.

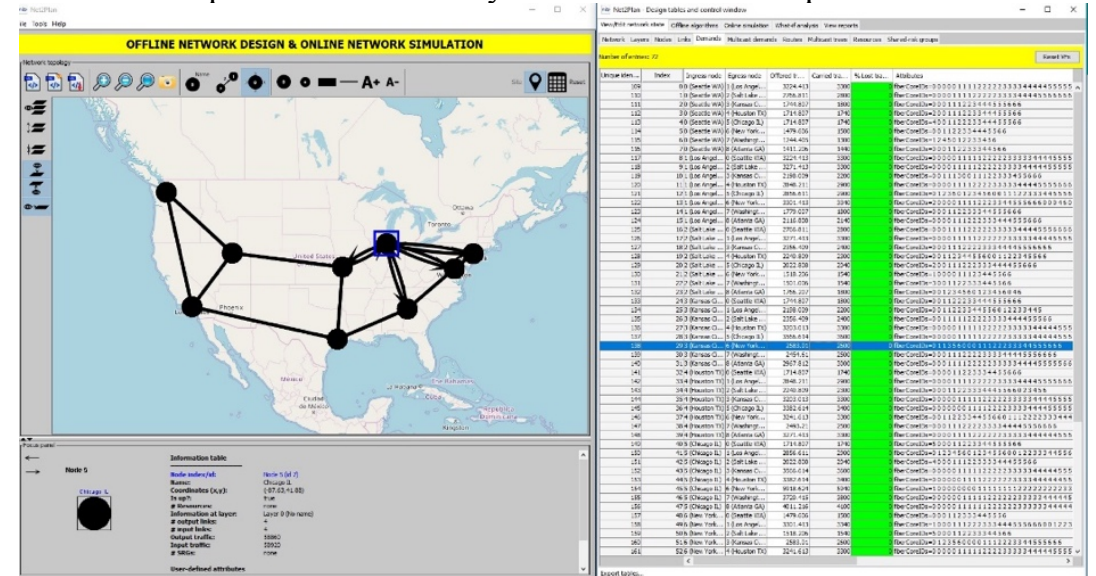

Figure 1. Net2plan framework in a Flex-Grid/MCF scenario 
In [3] an illustrative use case of Net2plan as Flex-Grid/MCF framework is explained. This study is about the performance comparison of the FNB and CCC SDM-ROADMs by means of heuristics as well as benchmark ILP algorithms applied in two reference topologies (Internet2 and Top 7 Spain [1]). We assume an available FlexGrid spectrum of $120 \mathrm{FSs}$ of $12.5 \mathrm{GHz}, \mathrm{MCF}-\mathrm{enabled}$ network links with 7, 12, and 19 cores. For traffic modelling purposes, the offered IP traffic profile is assumed proportional to the nodes population and a set of
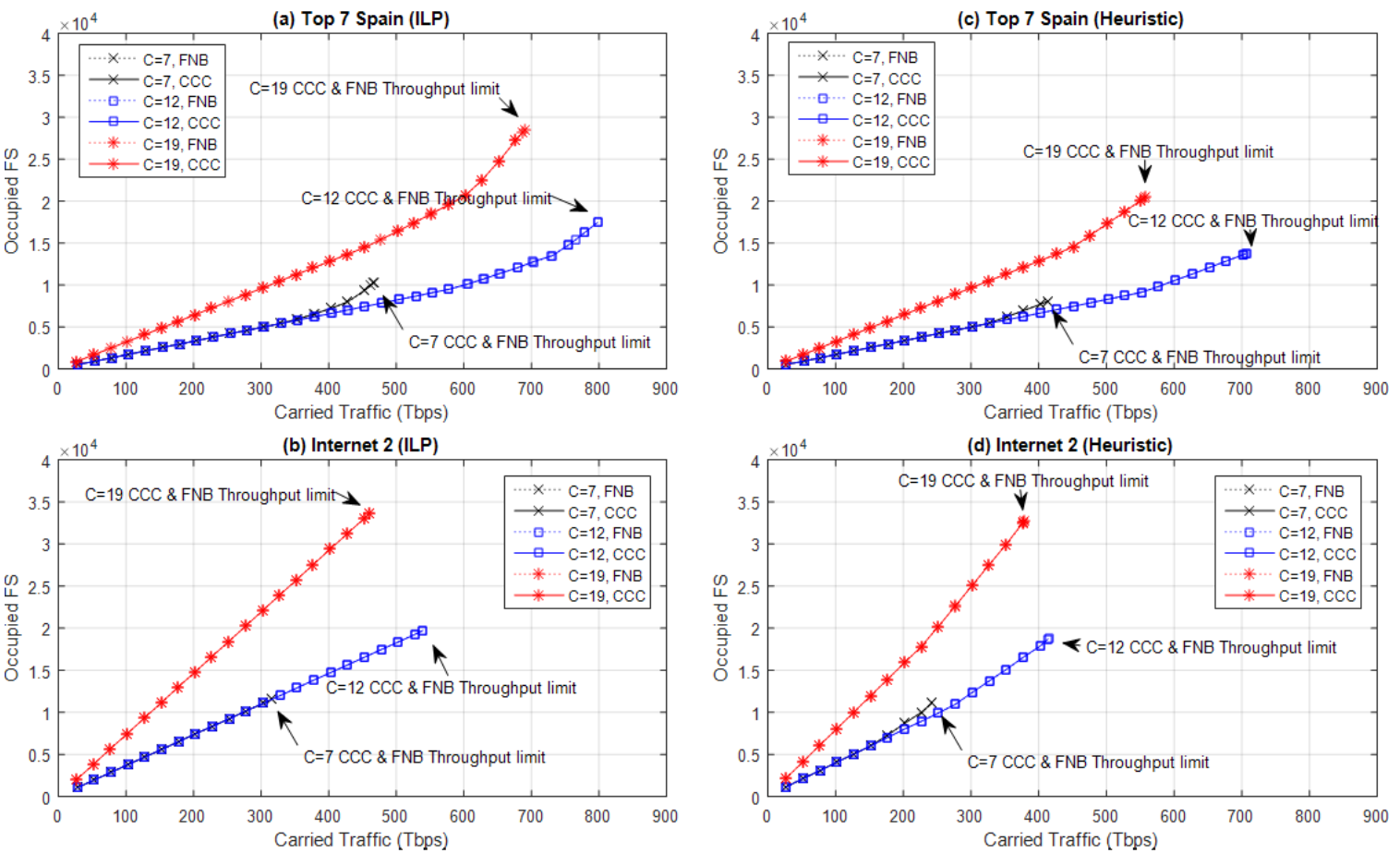

[1]

available transponders is defined to operate with 40, 100 and $400 \mathrm{Gbps}$ line rates, using different modulation formats (i.e., BPSK, QPSK, 16-QAM, 64-QAM). The algorithms select the modulation format based on the optical reach of its transponder. For each demand, $k=5$ shortest paths (w.r.t. distance in $\mathrm{km}$ ) are computed. Results in [3] and plotted in Fig. 2 describe the performance in terms of throughput of such scenario.

\section{PCE-BASED T-SDN CONTROLLER FOR PACKET OVER FLEXI-GRID OPTICAL NETWORKS}

A Transport SDN (T-SDN) controller is the responsible to provide the automatic control/configuration of connections within a transport network infrastructure. We consider a multi-layer network (MLN) combining both packet and optical (Flexi-Grid) switching technologies. Packet (MPLS) switching nodes are connected to Flexi-Grid optical switches (Bandwidth-Variable Optical Nodes, BV-OXCs) leveraging the benefits of the Sliceable Bandwidth Variable Transponders (SBVTs) [4]. This MLN allows exploiting the benefits of both worlds: the statistical multiplexing and finer granularity provided by packet switching, and the huge transport capacity and optical spectral efficiency of optical Flexi-Grid.

Figure 1 depicts the considered MLN where packet nodes (A, B, C and D) are connected to SBVTs bound to particular BV-OXCs. Packet network elements (NEs) are equipped with $p$ client ports operating at a given data rate. In the example client ports $(p: 1)$ operate at $400 \mathrm{~Gb} / \mathrm{s}$. SBVTs are made up of a pool of $N$ sub-transponders allowing generating up to $N$ different optical flows. Each optical flow occupies an optical frequency slot (FS) which is determined by a central frequency (e.g., $193.1 \mathrm{GHz}$ ) and a slot width (e.g. multiple of $12.5 \mathrm{GHz}$ ). Every sub-transponder operates at a fixed symbol rate (e.g., 25 Gbauds) supporting different modulation formats (e.g., DP-QPSK, DP-8QAM, DP-16QAM). The selection of the modulation formats enables flexible data rates per optical flow depending on the maximum distance supported by each modulation format.

Packet connection requests ( $\mathrm{r} 1, \mathrm{r} 2$ and $\mathrm{r} 3$ in Fig. 3) dynamically arrive to the network demanding different bandwidth demands (e.g., 10, 40 and $100 \mathrm{~Gb} / \mathrm{s}$ ). The T-SDN controller is then the responsible for computing the path and configuring the MLN NEs to actually accommodate such a demand. The MLN path computation [5] adopts an on-line algorithm which aims at, as much as possible, optimizing the network resources in terms of packet ports, SBVT's sub-transponders and optical spectrum. This is attained fostering strategies and decisions for leveraging electrical and optical grooming opportunities. The basic principle of optical grooming is to allow offloading the electronic processing burden onto the optical layer. This also enhances the transponder and optical spectrum utilization (e.g., guard bands between neighbouring optical flows are removed). 


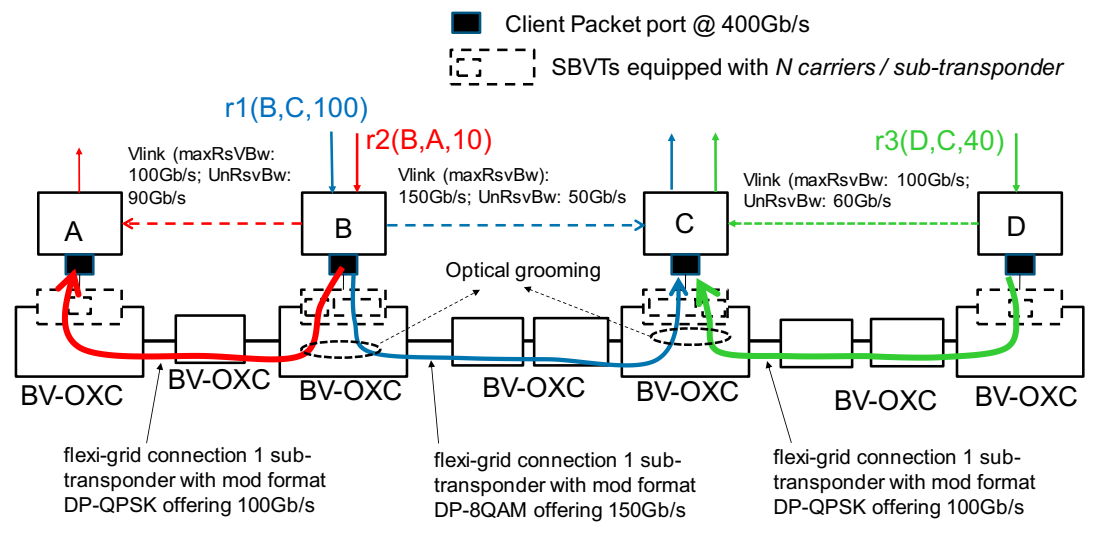

Fig. 3. MLN integrating packet and optical flexi-grid switches with SBVTs.

Figure 4.a) shows the implemented architecture of the PCE-based T-SDN controller for handling the MLN. The core building block is the Path Computation Element Central Controller (PCECC) being defined in the IETF which performs three basic functions: i) to process packet connections requests arriving via a NorthBound Interface (NBI) using a PCEP API. Each request specifies packet endpoints and bandwidth (b/s); ii) to perform path computation for received new packet demands specifying packet endpoints and required bandwidth (in $\mathrm{Gb} / \mathrm{s}$ ); iii) to handle the Provisioning Manager function for configuring NEs (packet and optical nodes as well as SBVTs) to set up/release packet and optical connections.

As mentioned above the Path Computation executes a devised MLN routing algorithm (Fig. 4.b). It uses the Traffic Engineering Database (TED) information (topology and network resources) updated by the Topology Manager (i.e., via BGP-LS with proposed extensions). The output of the Path Computation is encoded as $N$ (=> 1) Explicit Route Objects (EROs) describing the entire MLN path (i.e., nodes, links and network resources). One of these EROs is dedicated to the so-called Client Layer (packet) ERO which besides carrying the packet nodes and links contains the selected label (from the MPLS Label database). It is worth outlining that each packet connection uses the same common MPLS label in every path (physical or virtual) link. Each packet connection allocates a different MPLS label. If $\mathrm{N}>1$, the remaining EROs belong to the Server Layer (i.e. optical flexi-grid). Such EROs are related to optical path segments (nodes, links, FS and SBVTs). Each server layer connection induces a packet vlink. Both Client and Server Layer EROs are passed on to the Provisioning Manager function. This operates as an Active Stateful PCE storing existing (packet and optical) connections in the LSP database (LSPDB), and programming involved NEs via PCLabelUpd (Fig. 4.b).

To control each optical path segment, the Provisioning Manager relies on a standalone process referred to as Virtual Network Topology Manager (VNTM). VNTM is responsible for: i) retrieving link identifiers for the new virtual link. To do this, the VNTM communicates with the respective packet NE's Agents connected by the virtual link using a proprietary TCP protocol; ii) requesting to the Provisioning Manager (PCEP PCInitiate / PCRpt, the allocation/removal of optical resources such as frequency slot and SBVT (transceivers) specified in the Server Layer ERO; iii) triggering the virtual link creation (via the proprietary TCP protocol, including all its TE properties (metric and available bw) to the respective packet NEs' Agents. Finally, iv) VNTM occupies / releases the used bandwidth (reflecting the SBVT configuration) in the physical ports of the virtual link's NEs.

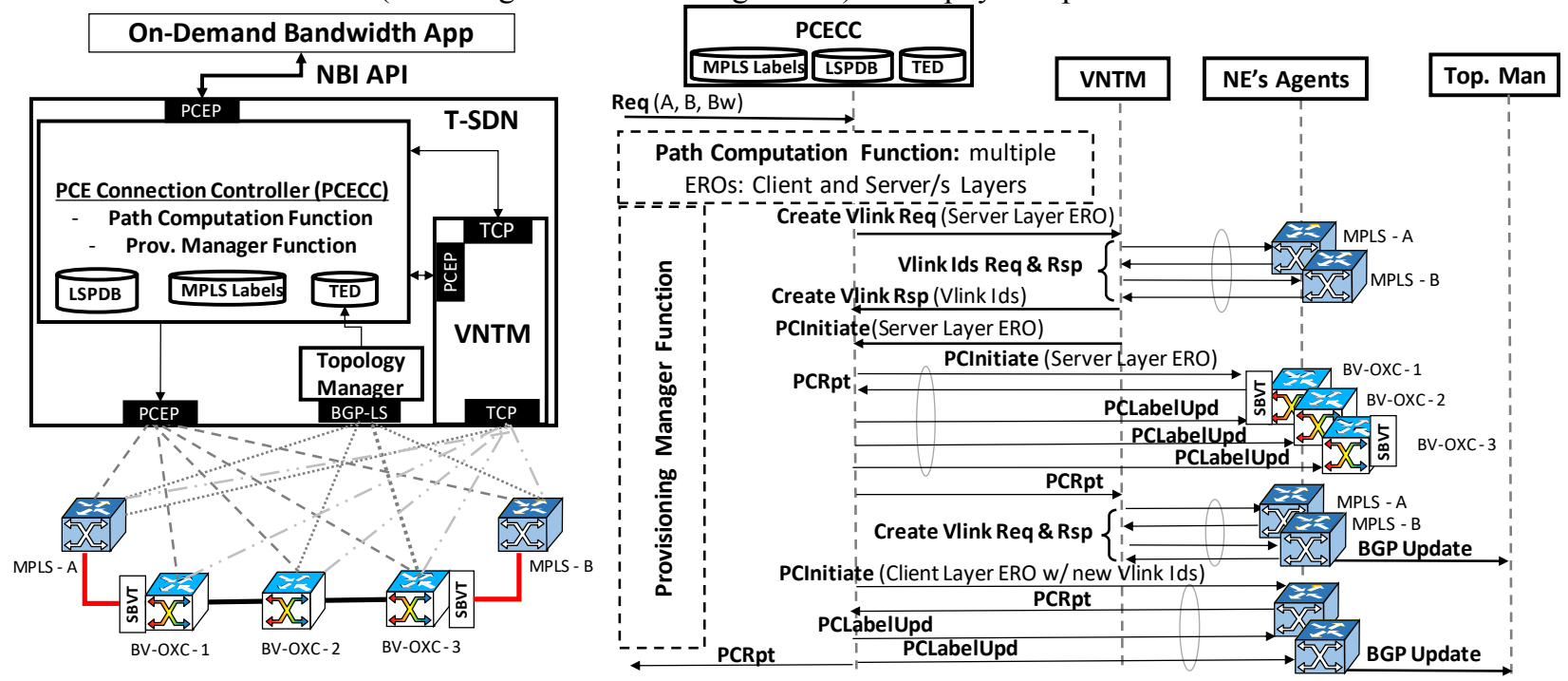

Fig. 4. a) PCE-based T-SDN Architecture for MLN; b) Workflow for setting up a packet connection 


\section{LIGHTPATH QOT ESTIMATION WITH AI TECHNIQUES}

One of the applications of Artificial Intelligence (AI) in optical networking is the estimation of lightpath Quality of Transmission (QoT) prior to deployment or set up. In dynamic networks, not only is the accuracy of the estimation crucial, but also the computation time, in order to handle lightpath requests in real-time. As a firststep towards the estimation of the QoT in Flex-Grid/SDM optical networks, we have proposed (e.g. [7]) the application of different supervised machine learning models to classify unestablished lightpaths into high or low QoT categories within impairment-aware $10 \mathrm{~Gb} / \mathrm{s}$ OOK wavelength-routed optical networks. The considered ML models include methods like support vector machines (SVM), logistic regression, classification and regression trees (CART), and random forests (RF). The QoT classifiers are trained by means of a training dataset, and 10fold cross-validation (CV) is used in order to select the best model and the tuning of its parameters. Fig 5. shows the results obtained (using R) when the Deutsche Telekom -DT- network is selected as physical topology and the spectrum of each fibre is divided into a fixed grid of 32 wavelength channels in terms of accuracy (Fig. 5.a) and prediction time (Fig. 5.b). The QoT threshold is set to $16.9 \mathrm{~dB}$, corresponding to a BER of $10^{-12}$. The results show accuracy is close to $99.9 \%$ (except for CART), with a very low prediction time.


Fig. 5 (a) Successful classification of lightpaths in high/low QoT categories in the DT network with 32 wavelength channels, (b) Prediction time of the different classification models.

The thematic network has also analysed the impact of combining different levels of flexibility in dynamic optical flexible backbone / metro networks: (i) with/out using slotted spectrum and (ii) allowing/not splitting the required capacity of a lightpath request over a set of sub-lightpaths together with traffic grooming. Moreover, different methods to solve the routing and spectrum assignment (RSA) problem have been proposed and analysed [8], as well as a techno-economic study of dynamic traffic grooming policies in elastic networks [9].

\section{ACKNOWLEDGEMENTS}

The authors would like to acknowledge the support of Elastic Networks (TEC2015-71932-REDT) project.

\section{REFERENCES}

[1] P. Pavon-Marino and J.-L. Izquierdo-Zaragoza: Net2Plan: an open source optical network planning tool for bridging the gap between academia and industry, IEEE Netw., vol. 29, no. 5, pp. 90-96, Oct. 2015.

[2] R. Rumipamba-Zambrano, et al.: Assessment of flex-grid/MCF optical networks with ROADM limited core switching capability, Proceedings of ONDM 2017, Budapest, May. 2017.

[3] F.-J. Moreno-Muro, et al.: Evaluation of Core-Continuity-Constrained ROADMs for Flex-Grid/MCF Optical Networks, IEEE/OSA J. Opt Commn. Netw., vol. 9, no. 11, pp. 1041-150, Nov. 2017."JOM - The Java Optimization Modeler" [Online]. Available: http://www.net2plan.com/jom.

[4] S. Zhang, et. al., "Evolving Traffic Grooming in Multi-Layer Flexible-Grid Optical Networks with Software-Defined Elasticity", IEEE/OSA Journal of Lightwave Technologies, vol. 32, no. 16, 2015.

[5] R. Martínez, et. al. "Experimental Evaluation of a PCE Transport SDN Controller for Dynamic Grooming in Packet over Flexi-Grid Optical Networks", in Proc. of ECOC 2017.

[6] J. Mata et al.: Artificial intelligence (AI) methods in optical networks: A comprehensive survey, Optical Switching and Networking, vol. 28. pp. 43-57, 2018. https://doi.org/10.1016/j.osn.2017.12.006

[7] J. Mata et al.: Supervised machine learning techniques for quality of transmission assessment in optical networks, in Proc. ICTON 2018.

[8] L. Ruiz et al.: Comparing Different Types of Flexibility when Solving the RSA Problem in EONs, in Proc. CSCI 2017.

[9] S. Fernández et al.: Techno-economic comparison of dynamic traffic grooming strategies for elastic optical networks, in Proc. ICTON 2017. 